\title{
On the Differential-Difference Properties of the Extended Jacobi Polynomials
}

\author{
By S. Lewanowicz
}

Abstract. We discuss differential-difference properties of the extended Jacobi polynomials

$$
P_{n}(x)={ }_{p+2} F_{q}\left(-n, n+\lambda, a_{p} ; b_{q} ; x\right) \quad(n=0,1, \ldots) .
$$

The point of departure is a corrected and reformulated version of a differential-difference equation satisfied by the polynomials $P_{n}(x)$, which was derived by Wimp (Math. Comp., v. 29, 1975, pp. 577-581).

1. Introduction. Here we are concerned with the properties of the extended Jacobi polynomials [3, Vol. 1, Section 7.4],

$$
\begin{aligned}
P_{n}(x) & ={ }_{p+2} F_{q}\left(\begin{array}{c}
-n, n+\lambda, a_{p} \\
b_{q}
\end{array}\right) \\
& =\sum_{k=0}^{n} \frac{(-n)_{k}(n+\lambda)_{k}\left(a_{p}\right)_{k}}{k !\left(b_{q}\right)_{k}} x^{k} \quad(n=0,1, \ldots),
\end{aligned}
$$

where

$$
(\alpha)_{k}=\Gamma(\alpha+k) / \Gamma(\alpha)
$$

is Pochhammer's symbol and the above contracted notation will be used throughout the paper:

$$
f\left(a_{p}\right)=\prod_{i=1}^{p} f\left(a_{i}\right), \quad f\left(b_{q}\right)=\prod_{j=1}^{q} f\left(b_{j}\right),
$$

$f$ being a given function. We assume that no $a_{i}$ equals any $b_{j}(i=1,2, \ldots, p$; $j=1,2, \ldots, q)$ and set

$$
b_{j}=1 \quad \text { for } j=q+1
$$

Let

$$
\boldsymbol{\sigma}=\max \{p+1, q\} .
$$

Wimp ([5]; see also [3, Vol. 2, Section 12.2]) has proved the following.

Received January 31, 1984.

1980 Mathematics Subject Classification. Primary 33A30. 
TheOREM 1.1. Let $\lambda, a_{i}, b_{j}(i=1,2, \ldots, p ; j=1,2, \ldots, q+1)$ be complex constants such that none of the quantities $\lambda, \lambda+1-b_{j}(j=1,2, \ldots, q)$ are negative integers or zero. Then the polynomials $P_{n}(x)$, see (1), satisfy the difference equation

$$
\sum_{m=0}^{\sigma+1}\left[C_{m}(n ; \sigma+1)+x D_{m}(n ; \sigma+1)\right] P_{n-m}(x)=0 \quad(n \geqslant \sigma+1),
$$

where

$$
C_{m}(n ; t)=\frac{(n-m+1)_{m}(2 n+\lambda-2 m)_{2 m}\left(n-m-1+b_{q+1}\right)}{m !(n+\lambda-m)_{m}(2 n+\lambda-m-t)_{m}\left(n-1+b_{q+1}\right)}
$$

$$
\begin{aligned}
& \times_{q+3} F_{q+2}\left(\begin{array}{c|c}
-m, 2 n+\lambda-m-t, n-m+b_{q+1} & \\
2 n+\lambda-2 m+1, n-m-1+b_{q+1} & 1
\end{array}\right), \\
& D_{m}(n ; t)=\frac{(n-m+1)_{m}(2 n+\lambda-2 m)_{2 m}\left(n-m+a_{p}\right)}{\Gamma(m)(n+\lambda-m)_{m}(2 n+\lambda-m-t+1)_{m-1}\left(n-1+b_{q+1}\right)}
\end{aligned}
$$

$$
\times_{p+2} F_{p+1}\left(\begin{array}{c|c}
1-m, 2 n+\lambda-m-t+1, n-m+1+a_{p} & \\
2 n+\lambda-2 m+1, n-m+a_{p} & 1
\end{array}\right) .
$$

Moreover, these polynomials do not satisfy a nontrivial equation of the form (4) of lower order than $\sigma+1$.

The next theorem supplements Theorem 1.1 in a certain particular case.

TheOREM 1.2 [2]. If $p+1=q$ and $x=1$ in (1) then the sequence $P_{n}(1)$ satisfies the recurrence relation

$$
\sum_{m=0}^{\sigma}\left[C_{m}(n ; \sigma)+D_{m}(n ; \sigma)\right] P_{n-m}(1)=0 \quad(n \geqslant \sigma) .
$$

The above result contains as a special case the recurrence relation given by Bailey [1] for

$$
{ }_{3} F_{2}\left(\begin{array}{c|c}
-n, n+\lambda, a_{1} & \\
b_{1}, b_{2} & 1
\end{array}\right)
$$

In this paper, our attention is focussed on another result of Wimp which is contained in the following theorem.

TheOrem 1.3 ([6]; see also [4, Section 5.13]). Let $\lambda \neq 1,2, \ldots$ and let the assumptions of Theorem 1.1 be satisfied. Then the polynomials $P_{n}(x)$ satisfy the differentialdifference equation

$$
x(\delta x-\varepsilon) \frac{d}{d x} P_{n}(x)=\sum_{m=0}^{\sigma}\left[A_{m}(n)+x B_{m}(n)\right] P_{n-m}(x),
$$


where

$$
\delta=\left\{\begin{array}{ll}
1 & (p+1 \geqslant q), \\
0 & (p+1<q),
\end{array} \quad \varepsilon= \begin{cases}0 & (p+1>q) \\
1 & (p+1 \leqslant q)\end{cases}\right.
$$

and

$$
\begin{aligned}
& A_{m}(n)=\left\{\begin{array}{lr}
\alpha_{m}(n)\left[\frac{1}{m !} \sum_{k=0}^{m} \frac{(-m)_{k}\left(n-k-1+b_{q+1}\right)}{k !(2 n+\lambda-k-\sigma)_{\sigma+1-m}}-\varepsilon\right] & (m>0) \\
\omega(n)-\varepsilon \alpha_{0}(n) & (m=0)
\end{array}\right. \\
& B_{m}(n)=\left\{\begin{array}{lr}
\alpha_{m}(n)\left[\delta-\frac{1}{\Gamma(m)} \sum_{k=0}^{m-1} \frac{(1-m)_{k}\left(n-k-1+a_{p}\right)}{k !(2 n+\lambda-k-\sigma)_{\sigma-m}}\right] & (m>0) \\
\delta \alpha_{0}(n) & (m=0)
\end{array}\right.
\end{aligned}
$$

and

$$
\alpha_{m}(n)= \begin{cases}(-1)^{m} \frac{(n-m+1)_{m}(2 n+\lambda-2 m)}{(n+\lambda-m)_{m}} & (m>0), \\ n & (m=0),\end{cases}
$$

$$
\omega(n)=\frac{n-1+b_{q+1}}{(2 n+\lambda-\sigma)_{\sigma}} .
$$

Moreover, no equation of the type (9) of lower order $\sigma^{\prime}<\sigma$ exists.

Note that the formulae (5) and (6) of the paper [6], defining the coefficients $A_{m}$ and $B_{m}$, respectively, are not correct as can be seen by considering the case of $p+1=q=2$ and $x=1$ and observing that the resulting second-order (pure) difference equation for quantities (8) disagrees with that given by Bailey [1]. An inspection of Wimp's proof of the equation (9) (see [6, esp. the last paragraph of Section II]) reveals how the formulae should be corrected.

Wimp's theorem was reproduced in the book [4] (see Theorem 2 in Section 5.13.2). Unfortunately, in the Russian edition of that book (Mir Publs., Moscow, 1980) the list of errors was increased by six other misprints.

2. Alternative Forms for $A_{m}$ and $B_{m}$. In Theorem 2.1, below, we give some alternative forms for the coefficients $A_{m}$ and $B_{m}$, see (1.11) and (1.12), respectively. We need the following lemma.

LEMMA 2.1. Let $m, r, s$ be integers $\geqslant 0$. Let none of the complex constants $\gamma, c_{i}$ $(i=1,2, \ldots, r)$ be integers. Then the identity

$$
\sum_{k=0}^{m} \frac{(-m)_{k}\left(c_{r}-k\right)}{k !(\gamma-s-k)_{s+1-m}}=(-1)^{m} \frac{(\gamma-2 m+1)_{2 m-1}\left(c_{r}-m\right)}{(\gamma-s-m)_{s+m}}
$$

$$
\times_{r+2} F_{r+1}\left(\begin{array}{c|c}
-m, \gamma-s-m, 1-m+c_{r} & \\
\gamma+1-2 m, c_{r}-m & 1
\end{array}\right)
$$

holds. 
Proof. Making use of some properties of Pochhammer's symbol [3, Vol. 1, Section 2.1], we obtain

$$
\begin{aligned}
(-1)^{m} & \frac{(\gamma-s-m)_{s+m}}{(\gamma-2 m+1)_{2 m-1}\left(c_{r}-m\right)} \sum_{k=0}^{m} \frac{(-m)_{k}\left(c_{r}-k\right)}{k !(\gamma-s-k)_{s+1-m}} \\
& =(-1)^{m} \sum_{k=0}^{m} \frac{(-m)_{k}(\gamma-s-m)_{m-k}\left(c_{r}-k\right)}{k !(\gamma-2 m+1)_{m-k}\left(c_{r}-m\right)} \\
& =(-1)^{m} \sum_{k=0}^{m} \frac{(-m)_{m-k}(\gamma-s-m)_{k}\left(c_{r}-m+k\right)}{(m-k) !(\gamma-2 m+1)_{k}\left(c_{r}-m\right)} \\
& =\sum_{k=0}^{m} \frac{(-m)_{k}(\gamma-s-m)_{k}\left(c_{r}-m+1\right)_{k}}{k !(\gamma-2 m+1)_{k}\left(c_{r}-m\right)_{k}} \\
& ={ }_{r+2} F_{r+1}\left(\begin{array}{c}
-m, \gamma-s-m, c_{r}-m+1 \\
\gamma-2 m+1, c_{r}-m
\end{array}\right) .
\end{aligned}
$$

THEOREM 2.1. The equations (1.11) and (1.12) can be rewritten in the form

$$
\begin{aligned}
& A_{m}(n)=\omega(n) C_{m}(n ; \sigma)-\varepsilon \alpha_{m}(n), \\
& B_{m}(n)=\omega(n) D_{m}(n ; \sigma)+\delta \alpha_{m}(n)
\end{aligned} \quad(m=0,1, \ldots, \sigma),
$$

respectively. Here the notation is that of (1.3), (1.5), (1.6), (1.10), (1.13) and (1.14).

Proof. Putting $r=q+1, \gamma=2 n+\lambda, s=\sigma, c_{i}=n-1+b_{i}(i=1,2, \ldots, q+1)$ in (1), we obtain

$$
\begin{aligned}
& \sum_{k=0}^{m} \frac{(-m)_{k}\left(n-k-1-b_{q+1}\right)}{k !(2 n+\lambda-\sigma-k)_{\sigma+1-m}} \\
& =(-1)^{m} \frac{(2 n+\lambda-2 m+1)_{2 m-1}\left(n-m-1+b_{q+1}\right)}{(2 n+\lambda-\sigma-m)_{\sigma+m}} \\
& \times_{q+2} F_{q+1}\left(\begin{array}{c|c}
-m, 2 n+\lambda-m-\sigma, n-m+b_{q+1} & \\
2 n+\lambda-2 m+1, n-m-1+b_{q+1} & 1
\end{array}\right) \\
& =\frac{m ! \omega(n)}{\alpha_{n}(n)} C_{m}(n ; \sigma) .
\end{aligned}
$$

Now, it readily follows that the first part of (1.11) may be written in the form (2). Obviously, the second part of (1.11) can be written as

$$
A_{0}(n)=\omega(n) C_{0}(n ; \sigma)-\varepsilon \alpha_{0}(n)
$$

because $C_{0}(n ; \sigma)=1$. Proceeding in a similar fashion, one arrives at (3).

Note that if $p+1=q$ and $x=1$ then the equation (1.9) takes the form (1.7) as, in view of (2) and (3), we have

$$
A_{m}(n)+B_{m}(n)=\omega(n)\left[C_{m}(n ; \sigma)+D_{m}(n ; \sigma)\right] .
$$

3. Further Differential-Difference Equations. With the aid of Theorems 1.3 and 2.1 we derive further differential-difference equations satisfied by the polynomials $P_{n}(x)$. We require one more lemma. 
LEMMA 3.1. We have

$$
\begin{aligned}
\text { (1) } & C_{m}(n ; \sigma)+\theta(n) C_{m-1}(n-1 ; \sigma) & =C_{m}(n ; \sigma+1) \quad(m=1,2, \ldots, \sigma+1), \\
\text { (2) } & C_{\sigma+1}(n ; \sigma) & =\varepsilon \alpha_{\sigma+1}(n) / \omega(n), \\
\text { (3) } & D_{m}(n ; \sigma)+\theta(n) D_{m-1}(n-1 ; \sigma) & =D_{m}(n ; \sigma+1) \quad(m=1,2, \ldots, \sigma+1), \\
\text { (4) } & D_{\sigma+1}(n ; \sigma) & =-\delta \alpha_{\sigma+1}(n) / \omega(n),
\end{aligned}
$$

where

$$
\theta(n)=\frac{n \omega(n-1)}{(n+\lambda-1) \omega(n)},
$$

and the notation used is that of (1.3), (1.5), (1.6), (1.10), (1.13) and (1.14).

Proof. Equations (1) and (3) can be checked by a straightforward calculation, using the definitions (1.5) and (1.6), respectively, and (5).

We prove the formula (2). We have

$$
C_{\sigma+1}(n ; \sigma)=\frac{(n-\sigma)_{\sigma+1}(2 n+\lambda-2 \sigma-2)_{2 \sigma+2}\left(n-\sigma-2+b_{q+1}\right)}{(\sigma+1) !(n+\lambda-\sigma-1)_{\sigma+1}(2 n+\lambda-2 \sigma-1)_{\sigma+1}\left(n-1+b_{q+1}\right)} f,
$$

where

$$
f={ }_{q+2} F_{q+1}\left(\begin{array}{c|c}
-\sigma-1, n-\sigma-1+b_{q+1} & \\
n-\sigma-2+b_{q+1} & 1
\end{array}\right)
$$

(see (1.5)). Now, observe that

$$
\begin{aligned}
f & =\frac{(-1)^{\sigma+1}(\sigma+1) !}{\left(n-\sigma-2+b_{q+1}\right)} & & (\sigma=q \geqslant p+1), \\
& =0 & & (\sigma=p+1>q)
\end{aligned}
$$

(cf. [3, Vol. 1, Section 2.9]). Thus, for arbitrary $p$ and $q$,

$$
\begin{aligned}
C_{\sigma+1}(n ; \sigma) & =\varepsilon(-1)^{\sigma+1} \frac{(n-\sigma)_{\sigma+1}(2 n+\lambda-\sigma)_{\sigma}(2 n+\lambda-2-\sigma)}{(n+\lambda-\sigma-1)_{\sigma+1}\left(n-1+b_{q+1}\right)} \\
& =\varepsilon \alpha_{\sigma+1}(n) / \omega(n) .
\end{aligned}
$$

Equation (4) can be proved in a similar way.

THEOREM 3.1. Under the assumptions of Theorem 1.3, the polynomials $P_{n}(x)$ satisfy the equations

$$
\begin{gathered}
x\left[\frac{d P_{n}(x)}{d x}+\frac{n}{n+\lambda-1} \frac{d P_{n-1}(x)}{d x}\right]=n\left[P_{n}(x)-P_{n-1}(x)\right] \\
x \frac{d P_{n}(x)}{d x}=\sum_{k=1}^{n}(-1)^{k} \frac{(-n)_{k}(2 n+\lambda-2 k)}{(1-\lambda-n)_{k}} P_{n-k}(x)+n P_{n}(x) .
\end{gathered}
$$


Proof. First observe that Eq. (1.9) can, by virtue of Theorem 2.1, be written in the form

$$
\begin{aligned}
x(\delta x-\varepsilon) \frac{d P_{n}(x)}{d x}= & (\delta x-\varepsilon) \sum_{m=0}^{\sigma} \alpha_{m}(n) P_{n-m}(x) \\
& +\omega(n) \sum_{m=0}^{\sigma}\left[C_{m}(n ; \sigma)+x D_{m}(n ; \sigma)\right] P_{n-m}(x) .
\end{aligned}
$$

Now, replace in the above equation $n$ by $n-1$, multiply the resulting equation by $n /(n+\lambda-1)$ and add to (8). The result is

(9)

$$
\begin{aligned}
& x(\delta x-\varepsilon)\left[\frac{d P_{n}(x)}{d x}+\frac{n}{n+\lambda-1} \frac{d P_{n-1}(x)}{d x}\right] \\
& =(\delta x-\varepsilon)\left[\sum_{m=0}^{\sigma} \alpha_{m}(n) P_{n-m}(x)+\frac{n}{n+\lambda-1} \sum_{m=1}^{\sigma+1} \alpha_{m-1}(n-1) P_{n-m}(x)\right] \\
& +\omega(n)\left\{\sum_{m=0}^{\sigma}\left[C_{m}(n ; \sigma)+x D_{m}(n ; \sigma)\right] P_{n-m}(x)\right. \\
& \left.+\theta(n) \sum_{m=1}^{\sigma+1}\left[C_{m-1}(n-1 ; \sigma)+x D_{m-1}(n-1 ; \sigma)\right] P_{n-m}(x)\right\} .
\end{aligned}
$$

Using Lemma 3.1 and considering that $C_{0}(n ; t)=1, D_{0}(n ; t)=0$, we write the right-hand side of (9) in the form

$$
\begin{aligned}
& (\delta x-\varepsilon) \sum_{m=0}^{\sigma+1} \beta_{m}(n) P_{n-m}(x) \\
& \quad+\omega(n) \sum_{m=0}^{\sigma+1}\left[C_{m}(n ; \sigma+1)+x D_{m}(n ; \sigma+1)\right] P_{n-m}(x),
\end{aligned}
$$

in which

$$
\beta_{m}(n)= \begin{cases}\alpha_{m}(n)+\frac{n}{n+\lambda-1} \alpha_{m-1}(n-1) & (m>0) \\ \alpha_{0}(n) & (m=0)\end{cases}
$$

or, in view of (1.13),

$$
\beta_{m}(n)= \begin{cases}n & (m=0) \\ -n & (m=1) \\ 0 & (m>1)\end{cases}
$$

By virtue of Theorem 1.1, the second sum of (10) is zero, therefore the right-hand side of (9) reduces to

$$
(\delta x-\varepsilon) n\left[P_{n}(x)-P_{n-1}(x)\right]
$$

and Eq. (6) follows. 
Formula (6) is a first-order difference equation with respect to $U_{n}(x)=x P_{n}^{\prime}(x)$. Using the well-known formula for the general solution of such an equation and remembering that $U_{0}(x)=0$ (see (1.1)), we get (7).

Of course, Eqs. (6) and (7) provide a generalization of the classical differential-difference formulae for the Jacobi polynomials, see [7, p. 262].

Introducing the notation

$$
Q_{n}(x)=(-1)^{n}(\lambda)_{n} P_{n}(x) / n !
$$

yields somewhat simpler forms of (6) and (7):

$$
\begin{gathered}
x\left[Q_{n}^{\prime}(x)-Q_{n-1}^{\prime}(x)\right]=n Q_{n}(x)+(n+\lambda-1) Q_{n-1}(x), \\
x Q_{n}^{\prime}(x)=\sum_{k=0}^{n-1}(2 k+\lambda) Q_{k}(x)+n Q_{n}(x) .
\end{gathered}
$$

Institute of Computer Science

University of Wrockaw

51-151 Wrockaw, Poland

1. W. N. Bailey, "Contiguous hypergeometric functions of the type ${ }_{3} F_{2}(1)$," Proc. Glasgow Math. Assoc., v. 2, 1954, pp. 62-65.

2. S. Lewanowicz, Recurrence Relations for Hypergeometric Functions of Unit Argument, Report N-127, Inst. Computer Sci., Univ. of Wroclaw, 1983. Also Math. Comp., v. 45, 1985. (To appear.)

3. Y. L. Luke, The Special Functions and Their Approximations, Academic Press, New York, 1969.

4. Y. L. LUKE, Mathematical Functions and Their Approximations, Academic Press, New York, 1975.

5. JeT WIMP, "Recursion formulae for hypergeometric functions," Math. Comp., v. 22, 1968, pp. 363-373.

6. JeT WimP, “Differential-difference properties of hypergeometric polynomials,” Math. Comp., v. 29, 1975. pp. 577-581.

7. E. D. Rainville, Special Functions, Macmillan, New York, 1960. 